\section{P3.423 ESTIMATING THE IMPACT OF ANTIRETROVIRAL THERAPY AND CONDOMS IN THE HIV EPIDEMIC OF BOGOTA, COLOMBIA}

doi:10.1136/sextrans-2013-051184.0874

J F Vesga, M C Boily, T B Hallett. Department of Infectious Disease Epidemiology, School of Public Health, Imperial College London, London, UK

Background The HIV epidemic in Bogota, Colombia is mainly driven by transmission among men-who-have-sex-with-men (MSM). Combined Antiretroviral Therapy (ART) introduced since 1997 is hypothesised to have helped control the overall spread of HIV. Our objective was to estimate the impact of ART on HIV incidence in the general population of Bogota.

Methods We developed a deterministic mathematical model simulating the sexual transmission of HIV among MSM, female-sexworkers, their clients and heterosexuals. Parameter values for behaviours and intervention coverage reflect local sources as much as possible. Surveillance data reflect a marked increase in condom use among FSW and MSM after the AIDS era- $80 \%$ and $59 \%$ in 2010, respectively-0. The model was fitted to FSW and MSM HIV prevalence and AIDS-mortality estimates using a rejection-sampling based approach that was used to create inferences on the scale of changes in risk caused by interventions. Counterfactual scenarios were generated in which the inferred impact of changes in condom use and ART were removed.

Results Between 1997 and 2011, there was 32,864 new infections and $\sim 9,500$ AIDS deaths, more than $80 \%$ of which were among MSM. The model estimates that there would have been an additional $\sim 96,860$ new infections and $\sim 17,353$ AIDS deaths if condom use has not increased over time. In the same period, ART became available, without which there may have been a further additional $\sim 4,060$ new infections and $\sim 4,493$ deaths. Future projections with levels of condom use and ART remaining constant, however, do not show further declines in HIV incidence.

Conclusions Antiretroviral-therapy and condom use may have had a major impact in curbing the HIV epidemic in Bogota. Further reductions in the future require additional prevention efforts focused on men-who-have-sex-with-men. The strength of these conclusions, however, is moderated by incomplete data on changing patterns of risk behaviour in this population.

\section{P3.424 TESTING TOGETHER: HIV/STI TESTING AND DIAGNOSES AMONG MALE COUPLES, CHICAGO, 2011-2012}

doi:10.1136/sextrans-2013-051184.0875

\begin{abstract}
${ }^{1,2,3}$ B Gratzer, ' $\mathrm{K}$ Cates, ${ }^{4} \mathrm{R}$ Stephenson, ${ }^{4} \mathrm{P}$ Sullivan, 'J Kane, 'S Hoehnle. 'Howard Brown Health Center, Chicago, IL, United States; ${ }^{2}$ Ann \& Robert H. Lurie Children's Hospital of Chicago, Chicago, IL, United States; ${ }^{3}$ University of Illinois at Chicago-School of Public Health, Chicago, IL, United States; " $E$ Emory University Rollins School of Public Health, Atlanta, GA, United States
\end{abstract}

Background Couples-based HIV/STI interventions for MSM are rare although approximately two-thirds of HIV transmission occurs in primary sexual dyads. We adapted and evaluated a couples voluntary counselling and testing intervention used with heterosexuals in Africa for MSM in the U.S.

Methods Between September 2011 and November 2012, 177 male couples (354 individuals) provided consent and were enrolled in "Testing Together" at two Chicago clinics. Demographics and clinical data were abstracted from electronic medical records and an online survey. Results Median age was 29 and a significant percentage (44\%) was aged $25-34$. 60\% were non-Hispanic White, 21\% Hispanic, and $8 \%$ Black/African-American. $34 \%$ had been together for less than three months, and $69 \%$ were together less than a year. Twenty-three par- ticipants $(6.5 \%)$ tested HIV positive. Four individuals reported a previous positive HIV result and may have used to the service to disclose their status to their partner, yielding 19 (5.4\%) newly identified infections. Five couples were concordantly positive $(2.8 \%)$ and nine were discordant (5.1\%). All four previously positive participants had discordantly negative partners. $64 \%$ of individuals (227) also tested for syphilis and five new syphilis infections were diagnosed $(2.2 \%)$; one couple was concordantly newly infected. Only $27 \%$ of individuals (95) tested for gonorrhoea and Chlamydia at any anatomic site. Of these, 10 were diagnosed with gonorrhoea $(10.5 \%)$ and 9 were diagnosed with Chlamydia (9.5\%); one couple was concordantly positive for each gonorrhoea and Chlamydia.

Conclusions Diagnoses of all infections was high, suggesting that Testing Together effectively targeted high risk participants, who may not otherwise have received testing services. Receiving HIV/ STI results together may substantially reduce the risk of transmission within primary sexual dyads and enhance partner service outcomes. Identifying mechanisms to increase the proportion of participants receiving STI tests warrants attention. Further research and investment in couples-based interventions is needed.

\section{P3.425 POTENTIAL FOR INTERNET-BASED TESTING TO REACH GAY, BISEXUAL AND OTHER MEN WHO HAVE SEX WITH MEN (MSM) IN CANADA FACING CURRENT BARRIERS TO TESTING FOR HIV AND SEXUALLY TRANSMITTED INFECTIONS}

doi:10.1136/sextrans-2013-051184.0876

${ }^{1,2} \mathbf{M}$ Gilbert, ${ }^{\top T}$ Salway Hottes, ${ }^{3} \mathrm{~T}$ Trussler, ${ }^{3} \mathrm{R}$ Marchand, ${ }^{1} \mathrm{D}$ Taylor, ${ }^{4} \mathrm{C}$ Fairley, ${ }^{5} \mathrm{~T}$ Wong, 'R Lester, 'G Ogilvie, ${ }^{2} \mathrm{~J}$ Shoveller. ${ }^{1}$ British Columbia Centre for Disease Control, Vancouver, BC, Canada; ${ }^{2}$ School of Population and Public Health, University of British Columbia, Vancouver, BC, Canada; ${ }^{3}$ Community Based Research Centre, Vancouver, $B C$, Canada; "Melbourne School of Population and Global Health, University of Melbourne, Melbourne, Australia, ${ }^{5}$ Public Health Agency of Canada, Ottawa, ON, Canada

Background While pilot studies indicate MSM will use internet testing, no known studies have examined the acceptability of internet testing among diverse MSM. We investigated intention to use internet testing for HIV/STIs, perceived benefits, and drawbacks in the 2011 Sex Now online survey of MSM in Canada.

Methods Participants were recruited through websites (76\%), organisations $(10 \%)$, and word-of-mouth $(9 \%)$. Intention to use was measured through a 5 -point Likert scale. We used multivariable logistic regression (comparing likely or very likely vs other options combined) to identify factors associated with intent (spanning socio-demographics, sexual behaviours, healthcare access, and internet use).

Results Among 7938 Canadian participants, 72\% intended to use internet testing with little variation across sub-groups. In multivariable analysis, intent to use was significantly higher $(p<0.05)$ for: younger age, less formal education, not being 'out' about sexuality, finding sex partners/searching for health information online, greater mobile phone use, faster uptake of new technologies generally, recent unprotected anal sex with a partner of unknown/discordant HIV status, HIV-negative status, no recent HIV/STI test, avoiding or delaying testing because of privacy concerns or difficulty with access, and dissatisfaction with healthcare services in general. Among men intending to use, most common perceived benefits were greater privacy $(32 \%)$, convenience $(24 \%)$, immediacy $(13 \%)$, and not seeing a nurse/doctor (12\%). Among men not intending, most common drawbacks were not seeing a nurse/doctor $(20 \%)$, wanting to talk to a provider in person about results $(17 \%)$, not wanting online results (18\%), and low trust of service (15\%).

Conclusions While all groups of Canadian MSM reported high intention to use internet-based testing, intent was higher for men 